\title{
Evaluation of the quality of Zernike phase plates
}

\author{
Michael Marko ${ }^{1,2}$, Xing Meng ${ }^{1}$, Chyongere Hsieh ${ }^{1}$, Gregory Kishchenko ${ }^{1}$ and ArDean Leith ${ }^{1}$ \\ 1. Wadsworth Center, New York State Department of Health, Empire State Plaza, Albany, NY 12201 \\ 2. College of Nanoscale Science and Engineering, 251 Fuller Rd., Albany, NY 12203
}

Thin-film phase plates, which usually have a central hole for the unscattered beam, are made of various materials, using various methods [1,2]. For optimal use, they should be evaluated for (1) absence of charging, (2) correct phase shift, and (3) cut-on frequency. During imaging at the TEM, centering of the phase plate (PP) needs to be maintained, and post-processing of the images may often be desirable.

Phase-plate quality is best evaluated by studying the power spectra ("FFT") of images of an amorphous film. We find that the thick carbon films of the commercial cryo-TEM grids that have a regular array of holes are good test specimens. It is convenient to record these images at an accurately known underfocus of about one micrometer. Applications such as CTFFIND [3] can be used to calibrate the defocus setting of the TEM, employing power spectra of images of the amorphous film recorded without using a PP.

The phenomenon of PP charging, while not completely understood, is frequently observed, especially as phase plates "age" $[4,5]$. Severe charging results in obvious distortion of the power spectrum (Fig. 1A), while less-severe charging may result in a subtle change such that the power spectrum does not correspond to the CTF which would be expected from the known electron optical parameters (Fig. 1B) [6].

The phase shift can be measured by noting the positions of the minima in a power spectrum of a defocused amorphous-film image, and then comparing them to the zeros in the calculated CTF (Fig. 2). Radostin Danev has made an interactive Excel spreadsheet for calculating and plotting PP CTF, which can be forwarded on request (mike.marko.em[at]gmail.com). One can also approximate the phase shift of a standard $\pi / 2\left(90^{\circ}\right)$ PP by changing the CTF from sin to cos.

The cut-on frequency is the spatial frequency below which the PP does not apply a phase shift. It is determined by the size of the central hole in the phase plate, the objective-lens focal length, and the acceleration voltage, although it is best to directly measure the cut-on from the power spectrum, as shown in Figure 3.

The centering of the PP on the unscattered beam can be monitored by adjusting the final condenser lens to an "off-plane" condition so that the illumination crosses over above or below the objective-lens back focal plane. This makes the shadow of the central hole visible in the image plane [6]. The shadow of the PP central hole expands to fill the image filed when the condenser is set to the "on-plane" condition. The PP is centered if the shadow of the hole expands symmetrically as the condenser is adjusted toward the onplane condition. However, minor mis-centering is more conveniently assessed by simply observing the image for asymmetric fringes around particles such as gold fiducial markers. We are developing algorithms to measure the magnitude and direction of the mis-centering so that the phase-plate can automatically be centered. We are also working on algorithms to correct the image for minor miscentering. Finally, a ringing effect (fringes) in the image is caused when the central hole has a sharp cut-on 
$[7,8]$. This can cause misinterpretation of the data in a 3-D reconstruction, and should be corrected; denoising and spatial-frequency filtering may also be beneficial in 3-D reconstruction procedures [9].

\section{References}

[1] M Marko et al., J. Struct. Biol. 184 (2013), p. 237.

[2] R Danev and K Nagayama, Ultramicroscopy 88 (2001), p. 243.

[3] J Mindell and N Grigorieff, J Struct. Biol. 142 (2003), p. 334.

[4] R Danev et al., Ultramicroscopy 109 (2009), p. 312.

[5] RM Glaeser et al., Ultramicroscopy 136 (2913), p. 6.

[6] M Marko et al., J. Struct. Biol. 174 (2011), p. 400.

[7] Y Fukuda et al., J. Struct. Biol. 168 (2009), p. 476.

[8] R Danev et al., Ultramicroscopy 111 (2011), p. 1305.

[9] H Sui et al., These proceedings (2014).

[10] Supported by NIH grant GM103555 (M. Marko).
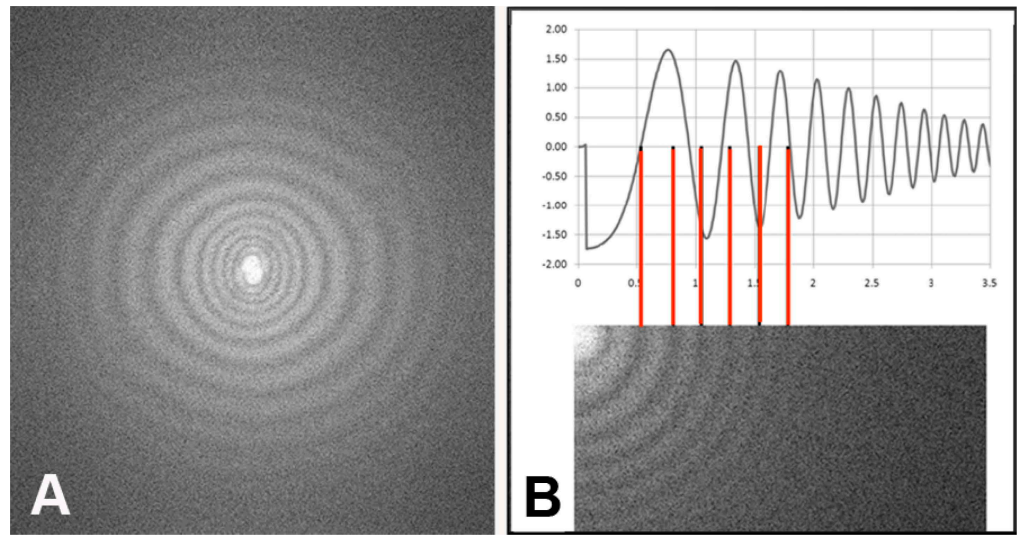

FIG. 1. Phase-plate charging. A. Severe charging--spacing between minima increases instead of decreases; cut-on appears "double" as from a mis-centered PP. B. Moderate "CTF distortion"--minima are evenly spaced, not matching the calculated CTF (from [6]).

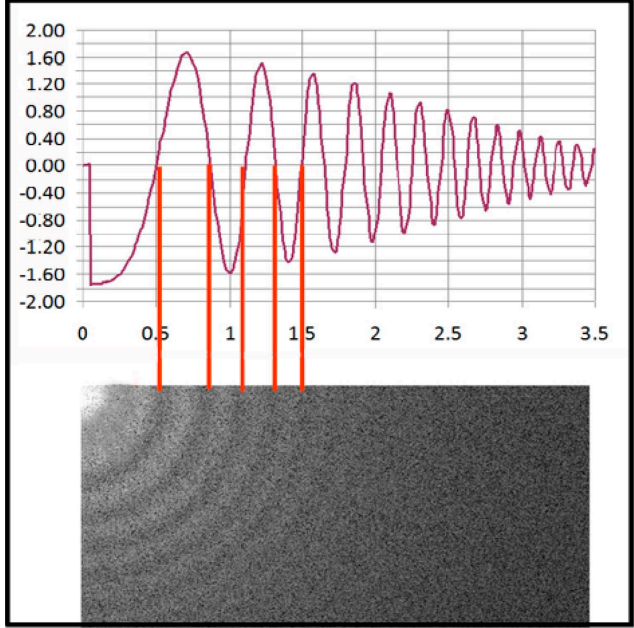

FIG. 2. Phase shift measured by comparing to a calculated CTF: Example shows a correct $\pi / 2$ shift.

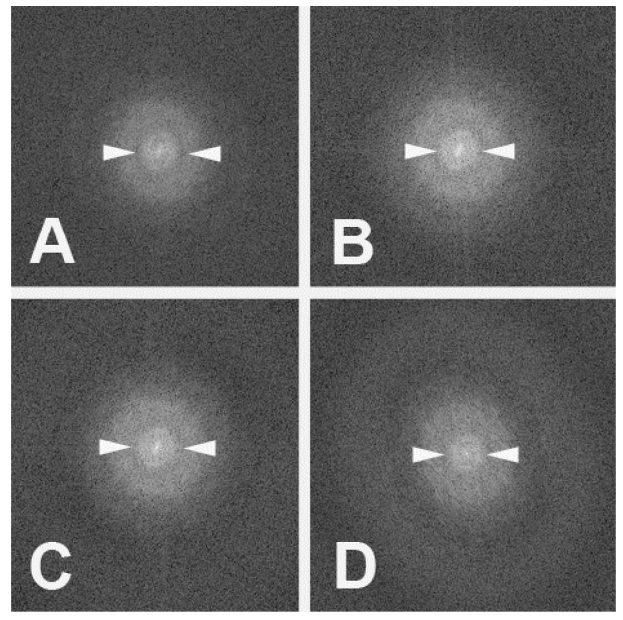

FIG. 3. Cut-on frequency determination: Magnified center of power spectra; A,B near focus; C,D $\Delta \mathrm{f} \sim-1 \mu \mathrm{m}$. Radius $\sim 25$ pixels, thus $\mathrm{d}=30 \mathrm{~nm}$ under conditions used. Astigmatism in D hardly affects cut-on. 